\title{
UTILIZAÇÃO DOS JOGOS REDUZIDOS NO ENSINO DO HANDEBOL: A INFLUÊNCIA NAS AÇÕES TÁTICAS
}

Filipe Manuel Clemente, Universidade de Coimbra - FCDEF-UC, Coimbra - Portugal Rúben Filipe Rocha, Universidade de Coimbra - FCDEF-UC, Coimbra - Portugal

\section{RESUMO}

O presente estudo objetivou analisar a influência de dois espaços diferenciados de prática na consecução de ações táticas de alunos durante a exercitação de formas de jogo reduzidas de handebol. Participaram no estudo oito alunos do género masculino $(18,25 \pm 1,04$ anos de idade). Os resultados evidenciam diferenças estatisticamente significativas no que se refere aos indicadores de número de ataques $\left(t_{(4)}=4,811 ; p\right.$-value $\left.=0,009\right)$ e número de ataques organizados $\left(t_{(4)}=6,364 ; p\right.$-value $\left.=0,003\right)$. Através do presente estudo foi possível comprovar a pertinência da manipulação de constrangimentos da tarefa relacionados com o espaço, influenciando a concretização de ações táticas desempenhadas pelos alunos.

Palavras-Chave: Educação Física; Handebol; Jogos reduzidos; Tática.

\section{USING SMALL-SIDED GAMES IN THE HANDBALL TEACHING: THE INFLUENCE IN TACTICAL ACTIONS}

\begin{abstract}
The aim of this study was to analyze the influence of two different spaces of practice in the students' tactical actions during the handball small-sided games. Participated in the study eight male students (18.25 \pm 1.04 years old). The results shows statistical differences in the number of attacks $(\mathrm{t}(4)=4,811 ; \mathrm{p}$-value $=0,009)$, as well in the number of organized attacks $(\mathrm{t}(4)=6,364 ; \mathrm{p}$-value $=0,003)$. Through this study was possible to confirm the pertinence of space task constraints, influencing the tactical actions performed by the students.
\end{abstract}

Key-Words: Physical Education; Handball; Small-sided games; Tactics. 


\section{INTRODUÇÃO}

Fatores fisiológicos, psicológicos, sociais, técnicos e táticos concorrem para a definição da aprendizagem e rendimento desportivo, ${ }^{1}$ através da sua interação constante ao longo do tempo. Consequentemente, a conceção da sessão de prática deverá considerar simultaneamente esses fatores de forma a desenvolver, de forma integral e corretamente adequada, os praticantes. ${ }^{2}$ Nesse sentido, a literatura demonstra relativa consensualidade no que respeita à estimulação dos praticantes através de exercícios que emulem situações contextuais da modalidade abordada. ${ }^{3,4,5,6}$ Assim, de forma a conferir realismo às tarefas os professores procuram adaptar os constrangimentos da tarefa no sentido de redirecionar a prática aos conteúdos, mantendo a ecologia característica da modalidade. ${ }^{7,8,9}$ Desta forma os constrangimentos da $\operatorname{tarefa}^{10}$ consubstanciam-se como elementos de indubitável pertinência para o processo de ensino desportivo. Efetivamente, os constrangimentos impostos pelo professor permitem afinar percetivamente os alunos com os objetivos da tarefa, bem como, potenciam a realização de determinadas ações. ${ }^{11,12,13}$

Recorrendo a tarefas ecológicas aproximar-se-ão as condições de prática ao realismo da modalidade, no sentido de promover um efetivo transfer para situações de jogo, através do desenvolvimento de fatores tático/técnicos potenciando o momento da exercitação ${ }^{14}$. Comummente, a literatura designa essas adaptações ao formato original dos jogos desportivos coletivos como jogos reduzidos (tradução do termo small-sided games). Assim, os jogos reduzidos são geralmente utilizados de forma a desenvolver aprendizagens ou parâmetros da performance de forma simultânea, enquadrando-os em tarefas ecológicas que emulem determinada especificidade do jogo. ${ }^{15,16,17}$

Constrangimentos relacionados com o espaço, número de jogadores e tempo de exercitação revelam-se como fatores fundamentais para a estruturação das tarefas de ensino. ${ }^{11}$ Efetivamente a opção por jogos reduzidos reduzem as possibilidades de passividades dos praticantes, aumentando os contactos da bola por indivíduo, resultando, desta forma, no incremento da consumação das ações ofensivas e defensivas de jogo. ${ }^{18}$ Assim, a manipulação das dimensões do campo e do número de jogadores por tarefa poderão repercutir-se na intensidade da prática, ${ }^{19,20,21,22}$ bem como, em variáveis técnico/táticas concretizadas pelos alunos. ${ }^{23}$ 
Face ao exposto, o presente trabalho objetiva analisar a manipulação de constrangimentos espaciais no desenvolvimento de conteúdos táticos concretizados pelos alunos em diferentes formas de jogo de handebol, procurando verificar possíveis influências que o espaço de prática origina na ação dos alunos.

\section{MÉTODOS}

\section{Amostra}

Participaram no estudo oito alunos do género masculino $(18,25 \pm 1,04$ anos de idade). Todos os alunos participaram voluntariamente, assinando um termo de consentimento livre e esclarecido. O estudo foi realizado em conformidade com o código de ética da Universidade de Coimbra e as recomendações da Declaração de Helsínque em Pesquisa com Seres Humanos. Os participantes não sofriam de qualquer tipo de incapacidade física ou mental.

\section{Tarefa}

A tarefa consistiu em transportar a bola da zona defensiva até à zona ofensiva da equipa adversária, respeitando os princípios de jogo e regras da modalidade, ultrapassando a linha de baliza adversária através de um passe para um atacante que recebe a bola após a linha de baliza da equipa adversária ("zona de ponto" da sua equipa).

a)

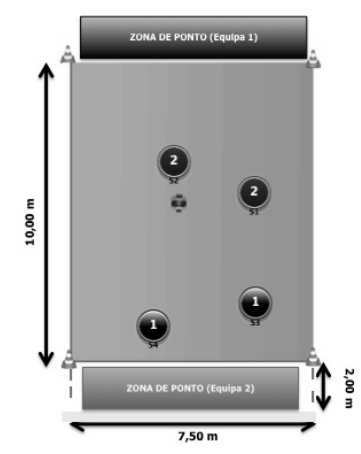

b)

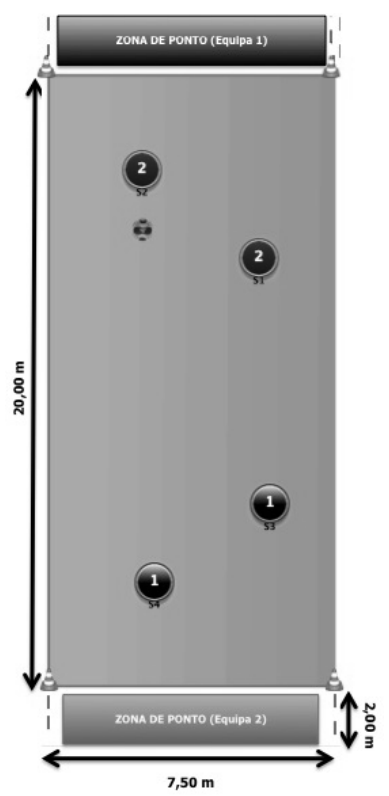

Figura 1: Espaços de prática manipulados. 
A tarefa decorreu durante cinco minutos, onde o principal objetivo era assumido pela marcação de um ponto por cada transposição válida (i.e., receção da bola em trajetória aérea por um único elemento da equipa atacante após a linha de baliza da equipa adversária, na zona de ponto da sua equipa).

\section{Instrumentos}

Para a filmagem das ações dos alunos utilizou-se uma câmara digital SLR (Canon EOS 500D) com capacidade para processar imagens a $30 \mathrm{~Hz}$ (i.e., 30 imagens por segundo). Esta foi colocada a $4,53 \mathrm{~m}$ acima do solo, no plano sagital à realização da tarefa. Utilizaram-se bolas regulamentadas pela Federação Portuguesa de Andebol para a faixa etária dos alunos. Coletes azuis e verdes foram utilizados de forma a diferenciar as equipas em confronto. A análise notacional de jogo realizou-se através da visualização posterior das filmagens, recorrendo a documentos de observação concebidos para o efeito.

\section{Procedimentos}

O primeiro espaço (1/8 do espaço formal) compreendeu uma área de 10 x 7,5 metros e o segundo espaço (2/8 do espaço formal) uma área de 20 x 7,5 metros Paralelamente à área da linha de baliza, prolongava-se em 2 metros de comprimento uma área (zona de ponto) que definia a zona de receção da bola por parte do atacante.

A tarefa decorreu em seis situações distintas, interagindo as formas de jogo 2x2, 3x3 e 4x4 com os espaços de 1/8 e 2/8 do campo formal.

\section{RESULTADOS}

Apresentar-se-ão de seguida os indicadores táticos que provêm do comportamento coletivo no que se refere à consecução dos objetivos de jogo. No sentido de simplificar o processo de apresentação das tabelas utilizar-se-ão as siglas de E. para o espaço de prática, bem como, 1/8 (espaço inferior) e 2/8 (espaço superior). 
Tabela 1- Conteúdos Táticos (Eficácia dos Ataques Organizados e Contra-Ataques)

\begin{tabular}{|c|c|c|c|c|c|c|c|c|c|c|c|c|c|c|}
\hline \multirow{3}{*}{$\begin{array}{c}\text { Formas } \\
\text { de } \\
\text { Jogo }\end{array}$} & \multirow{2}{*}{\multicolumn{2}{|c|}{$\begin{array}{l}\text { Número de } \\
\text { Ataques }\end{array}$}} & \multicolumn{6}{|c|}{ Número de Ataques Organizados } & \multicolumn{6}{|c|}{ Número de Contra-ataques } \\
\hline & & & \multicolumn{2}{|c|}{ Total } & \multicolumn{2}{|c|}{ Sem Golo } & \multicolumn{2}{|c|}{ Com Golo } & \multicolumn{2}{|c|}{ Total } & \multicolumn{2}{|c|}{ Sem Golo } & \multicolumn{2}{|c|}{ Com Golo } \\
\hline & E. $1 / 8$ & E. $2 / 8$ & E. $1 / 8$ & $\begin{array}{c}\text { E. } \\
2 / 8 \\
\end{array}$ & E. $1 / 8$ & $\begin{array}{c}\text { E. } \\
2 / 8\end{array}$ & E. $1 / 8$ & $\begin{array}{c}\text { E. } \\
2 / 8\end{array}$ & E. $1 / 8$ & $\begin{array}{c}\text { E. } \\
2 / 8\end{array}$ & E. $1 / 8$ & $\begin{array}{c}\text { E. } \\
2 / 8 \\
\end{array}$ & E. $1 / 8$ & $\begin{array}{c}\text { E. } \\
2 / 8\end{array}$ \\
\hline $2 \times 2$ & 29 & 26 & 24 & 20 & 15 & 14 & 9 & 6 & 5 & 6 & 0 & 0 & 5 & 6 \\
\hline $3 \times 3$ & 32 & 25 & 26 & 20 & 14 & 12 & 12 & 8 & 4 & 3 & 2 & 2 & 2 & 1 \\
\hline $4 \times 4$ & 31 & 23 & 26 & 18 & 18 & 8 & 8 & 10 & 4 & 4 & 1 & 1 & 3 & 3 \\
\hline
\end{tabular}

No que se refere ao indicador número de ataques, existem evidências estatísticas para se afirmar que a média no espaço $1 / 8$ é significativamente superior $\left(t_{(4)}=4,811 ; p\right.$-value $=$ $0,009)$.

Considerando o indicador número de ataques organizados, existem evidências estatísticas para se afirmar que a média no espaço $1 / 8$ é significativamente superior $\left(t_{(4)}=6,364\right.$; $p$ value $=0,003)$. Quanto ao número de ataques organizados como golo $\left(t_{(4)}=2,030 ; p\right.$-value $=0,112)$ e sem golo $\left(t_{(4)}=1,000 ; p\right.$-value $\left.=0,374\right)$ não existem evidências estatísticas que confirmem a sua variância.

No que se refere ao indicador número de contra-ataques, não existem evidências estatísticas para se afirmar que a média entre espaços de prática se diferencia significativamente $\left(t_{(4)}=0,000 ; p\right.$-value $\left.=1,000\right)$. Os mesmos resultados são verificados no que respeita ao indicador número de contra-ataques com golo $\left(t_{(4)}=0,000 ; p\right.$-value $=$ $1,000)$ e sem golo $\left(t_{(4)}=0,000 ; p\right.$-value $\left.=1,000\right)$.

Tabela 2 - Origem dos Pontos (Ataque Organizado ou Contra-Ataque) e Eficácia dos Ataques

\begin{tabular}{ccccccccc}
\hline & \multicolumn{3}{c}{ Origem dos Pontos } & \multicolumn{3}{c}{ Eficácia do Tipo de Ataque } \\
Formas & \multicolumn{2}{c}{ \% Ataque } & \multicolumn{2}{c}{$\%$ Contra- } & \multicolumn{2}{c}{$\%$ Ataque } & \multicolumn{2}{c}{$\%$ Contra- } \\
de Jogo & \multicolumn{2}{c}{ Organizado } & \multicolumn{2}{c}{ ataque } & \multicolumn{2}{c}{ Organizado } & \multicolumn{2}{c}{ ataque } \\
& E. & E. & E. & E. & E. & E. & E. & E. \\
& $1 / 8$ & $2 / 8$ & $1 / 8$ & $2 / 8$ & $1 / 8$ & $2 / 8$ & $1 / 8$ & $2 / 8$ \\
\hline $2 \times 2$ & 64 & 50 & 36 & 50 & 38 & 30 & 100 & 100 \\
$3 \times 3$ & 86 & 89 & 14 & 11 & 46 & 40 & 50 & 33 \\
$4 \times 4$ & 73 & 77 & 27 & 23 & 31 & 56 & 75 & 75 \\
\hline
\end{tabular}

Perspetivando os indicadores percentagem de origem dos pontos através do ataque organizado e contra-ataque, não existem evidências estatísticas para se afirmar que as 
médias entre espaços de prática se diferenciam significativamente $\left(t_{(4)}=0,176 ; p\right.$-value $=$ $0,869)$ e $\left(t_{(4)}=-0,176 ; p\right.$-value $\left.=0,869\right)$ respetivamente para ataque organizado e contraataque.

Quanto aos indicadores de eficácia do ataque organizado e contra-ataque, não existem evidências estatísticas para se afirmar que as médias entre espaços de prática se diferenciam significativamente $\left(t_{(4)}=-0,428 ; p\right.$-value $\left.=0,690\right)$ e $\left(t_{(4)}=0,229 ; p\right.$-value $=$ $0,830)$ respetivamente para ataque organizado e contra-ataque.

Tabela 3 - Marcações e Desmarcações concretizados pelos alunos

\begin{tabular}{ccccc}
\hline & & & & \\
$\begin{array}{l}\text { Formas } \\
\text { de Jogo }\end{array}$ & Marcações & \multicolumn{2}{c}{ Desmarcações } \\
& E. & E. & & \\
& $1 / 8$ & $2 / 8$ & E. $1 / 8$ & E. $2 / 8$ \\
\hline $2 \times 2$ & 65 & 56 & 35 & 36 \\
$3 \times 3$ & 75 & 71 & 52 & 54 \\
$4 \times 4$ & 78 & 69 & 60 & 73 \\
\hline
\end{tabular}

No que se refere ao indicador marcações individuais, não existem evidências estatísticas para se afirmar que a média entre espaços de prática se diferencia significativamente $\left(t_{(4)}=\right.$ $1,197 ;$-value $=0,298)$.

Quanto ao indicador desmarcações, não existem evidências estatísticas para se afirmar que a média entre espaços de prática se diferencia significativamente $\left(t_{(4)}=-0,411 ; p\right.$-value $=$ $0,702)$.

\section{DISCUSSÃO}

O espaço destinado à prática constituiu-se como um importante constrangimento da tarefa que poderá influenciar a perceção dos praticantes, bem como, a respetiva tomada de decisão e comportamento inerente. Nesse sentido, considerando que a tática é a interrelação dos fatores do jogo (e.g., espaço, tempo, colega, bola, adversário) na dependência direta do objetivo final da modalidade e dos objetivos táticos gerais e específicos da ação ${ }^{24}$, afigurando-se importante analisar a preponderância do espaço para a consecução do comportamento tático dos alunos. 
Face aos dados obtidos, é possível verificar que no espaço inferior (i.e., 1/8) existiu a maior frequência de ataques diferenciando-se estatisticamente do espaço superior (i.e., 2/8). Efetivamente, tal acontecimento poder-se-á associar ao menor espaço para percorrer no sentido de alcançar a linha de ponto, favorecendo a celeridade no processo de transição e, por consequência, incrementando o número de ações de ataque. Adicionalmente foi possível constatar que no espaço de prática inferior a frequência de ataques organizados diferenciaram-se estatisticamente do espaço superior sugerindo-se que o sucedido poderse-á relacionar com o reduzido espaço para concretizar o contra-ataque, com o maior número de marcações individuais, bem como, com o reduzido espaço de prática que diminui as oportunidades de desequilibrar o sistema defensivo adversário.

Igualmente importa verificar que no espaço de jogo $1 / 8$ a frequência de ações de desmarcação constituíram-se como inferiores relativamente ao espaço de prática superior (i.e., 2/8) sugerindo que o ataque organizado sucedia com menor dinamismo possivelmente originado pelo menor amplitude do espaço, bem como, pela menor profundidade reduzindo as oportunidades de desmarcação em espaços passíveis de originar o passe adequadamente concretizado.

No que se refere a ações de marcação, como exposto anteriormente, sucederam com maior frequência no espaço de jogo inferior possivelmente potenciadas pela maior proximidade em relação aos adversários incrementado pelo menor espaço passível de ser explorado pelos alunos em fase ofensiva. Dessa forma, os atacantes expor-se-iam de forma regular à marcação dos adversários devido à redução de oportunidades de tomada de decisão no que refere a movimentações de apoio ou rutura face ao companheiro com posse de bola. Nesse sentido, o menor espaço poderá reduzir o dinamismo ofensivo, incrementando as possibilidades dos defensores de efetivaram comportamentos táticos associados à fase referida. Inversamente, o incremento do espaço em profundidade despoletou a frequência de desmarcações, possibilitando a maior eficácia do ataque organizado em relação ao espaço inferior. Tal facto poderá ser justificado pelo menor número de marcações originado pela maior dificuldade em acompanhar as movimentações adversárias atacantes, bem como, pelo aumento das possibilidades de tomada de decisão tornando as ações ofensivas mais imprevisíveis e, consequentemente, com superior possibilidade de eficácia. 
Efetivamente, o espaço de prática possibilitará a maior ou menor perceção ou afinamento percetivo relativamente às possibilidades de ação, constrangendo desta forma as tomadas de decisão. ${ }^{11}$ Assim o acréscimo de espaço incrementará as possibilidades de movimentações procurando desequilibrar a defensiva adversária, i.e., potenciando o desenvolvimento dos alunos no que à construção e dinamismo coletivo ofensivo se refere. Desta forma, princípios de jogo como a mobilidade ofensiva ou a cobertura ofensiva poderão ser potenciados pelo incremento do espaço, favorecendo a perceção da eficácia de ações de desmarcação aos alunos. ${ }^{23}$ Inversamente, a redução do espaço de prática beneficiará a ação e consecução da fase defensiva, reduzindo a variabilidade ofensiva e, consequentemente, concedendo oportunidades de sucesso na ação do defensor, aproximando-o dos atacantes, bem como, posicionando-o em função da localização da bola. Igualmente possibilitará a consolidação de ações táticas defensivas, tais como, a cobertura defensiva ou a manutenção do equilíbrio defensivo.

\section{CONCLUSÃO}

O presente estudo demonstra a pertinência do espaço de prática como variável preponderante no momento de desenvolver os comportamentos táticos concretizados pelos alunos. $^{23}$ Tais constrangimentos da tarefa suscitam efeitos diferenciados no que ao comportamento tático se refere, pelo que, a manipulação de tais variáveis favorecerá a consecução dos princípios táticos definidos pelo professor. ${ }^{25}$ Consequentemente, o professor, aquando da determinação das tarefas de exercitação deverá refletir de forma cuidada sobre o espaço a definir para a prática, ${ }^{25}$ no sentido de adequar a exercitação aos conteúdos e objetivos previamente determinados, garantindo a consecução dos mesmos.

\section{REFERÊNCIAS}

${ }^{1}$ BANGSBO, J. The physiology of soccer with special reference to intense intermittent exercise. Acta Physiologica Scandinavica, v. 619, p. 1-155, 1994.

${ }^{2}$ JONES, S.; DRUST, B. Physiological and technical demands of $4 \mathrm{v} 4$ and $8 \mathrm{v} 8$ games in elite youth soccer players. Kinesiology, v. 39, n. 2, p. 150-156, 2007. 
${ }^{3}$ BOMPA, T. Theory and methodology of training. Dubusque: Kendall/Hunt, 1983.

${ }^{4}$ HELGERUD, J. et al. Aerobic endurance training improves soccer performance. Medicine and Science in Sports and Exercise, v. 33, n. 11, p. 1925-1931, 2001.

${ }^{5}$ AROSO, J.; REBELO, A. N.; GOMES-PEREIRA, J. Physiological impact of selected game-related exercises. Journal of Sports Sciences, v. 22, p. 522, 2004.

${ }^{6}$ MALLO, J.; NAVARRO, E. Physical load imposed on soccer players during small-sided training games. The Journal of Sports Medicine and Physical Fitness, v. 48, n. 2, p. 166-171, 2008.

${ }^{7}$ MACLAREN, D. et al. Physiological strain in 4-a-side soccer. In: REILLY, T. (Ed.). Science and Football. London: Taylor \& Francis, 1988. p. 76-80,

${ }^{8} \mathrm{HOFF}$, J. et al. Soccer specific aerobic endurance training. British Journal of Sports Medicine, v. 36, n. 3, p. 218-221, 2002.

${ }^{9}$ REILLY, T.; WHITE, C. Small-sided games as an alternative to interval-training for soccer players. In: REILLY, T.; CABRI, J.; ARAÚJO, D. (Ed.). Science and Football V. London: Routledge, 2004. p. 355-358.

${ }^{10}$ NEWELL, K. M. Constraints on the development of coordination. In: WADE, M. G.; WHITING, H. T. A. (Ed.), Motor development in children: aspects of coordination and control. Dordrecht: Martinus Nijhoff, 1986. p. 341-360.

${ }^{11}$ CLEMENTE, F.; MENDES, R. Aprender o jogo jogando: uma abordagem transdisciplinar. Revista Científica Exedra, v. 5, n. 1, p. 27-36, 2011.

${ }^{12}$ CLEMENTE, F; MENDES, R. Constrangimentos instrucionais em futebolistas sub-12 na sub-fase de jogo 1 x 1 com guarda-redes. In: MOUROÇO, P. (Ed.). Estudos em desenvolvimento motor da criança IV. Leiria: ESECS/IPL, 2011. p. 146-152.

Conexões: revista da Faculdade de Educação Física da UNICAMP, Campinas, v. 10, n. 2, p. 66-76, maio/ago. 2012.74 ISSN: 1983-9030 
${ }^{13}$ CLEMENTE F.; MENDES, R.; SOLER, F. C. Constrangimentos instrucionais em futebolistas sub-18 na sub-fase de jogo 1x1 com guarda-redes. Revista Portuguesa de Ciências do Desporto, v. 11, supl. 4, p. 45, 2011.

${ }^{14}$ WILLIAMS, A. M.; HORN, R.; HODGES, N. J. Skill acquisition. In: REILLY, T.; WILLIAMS, A. M. (Ed.). Science and Soccer. London: Routledge, 2003. p. 198-213.

${ }^{15}$ RAMPININI, E. et al. Factors influencing physiological responses to small-sided soccer games. Journal of Sports Sciences, v. 25, n. 6, p. 659-666, 2007.

${ }^{16}$ HILL-HAAS, S. et al. Variability of acute physiological responses and performance profiles of youth soccer players in small-sided games. Journal of Science and Medicine in Sport, v. 11, n. 5, p. 487-490, 2008.

${ }^{17}$ HILL-HAAS, S. V. et al. Physiological responses and time-motion characteristics of various small-sided soccer games in youth players. Journal of Sports Sciences, v. 27, n. 1, p. 1-8, 2009.

${ }^{18}$ BASTOS, M. J.; GRAÇA, A.; SANTOS, P. Análise da complexidade do jogo formal versus jogo reduzido em jovens do $3^{\circ}$ ciclo do ensino básico. Revista Portuguesa de Ciências do Desporto, v. 8, n. 3, p. 355-364, 2008.

${ }^{19}$ TESSITORE, A. et al. Physiological and technical aspects of "6-a-side" soccer drills. Journal of Sports Medicine and Physical Fitness, v. 46, n. 1, p. 36-43, 2006.

${ }^{20}$ CASAMICHANA, D.; CASTELLANO, J. Time-motion, heart rate, perceptual and motor behaviour demands in small-sides soccer games: Effects of field size. Journal of Sports Sciences, v. 28, n. 14, p. 1615-1623, 2010.

${ }^{21}$ KATIS, A.; KELLIS, E. Effects of small-sided games on physical conditioning and performance in young soccer players. Journal of Sports Science and Medicine, v. 8, p. 374-380, 2009.

Conexões: revista da Faculdade de Educação Física da UNICAMP, Campinas, v. 10, n. 2, p. 66-76, maio/ago. 2012.75 ISSN: 1983-9030 
${ }^{22}$ OWEN, A.; TWIST, C.; FORD, P. Small-sided games: the physiological and technical effect of altering field size and player numbers. Insight, v. 7, n. 2, p. 50-53, 2004.

${ }^{23}$ CLEMENTE, F. et al. The usefulness of small-sided games on soccer training. Journal of Physical Education and Sport, v. 12, n. 1, p. 93-102, 2012.

${ }^{24}$ BAYER, C. La ensenanza de los juegos desportivos colectivos. Espanha: Hispano Europea, 1986.

${ }^{25}$ CLEMENTE, F. Princípios Pedagógicos dos Teaching Games for Understanding e da Pedagogia Não-Linear no Ensino da Educação Física. Movimento, Porto Alegre, v. 18, maio 2012. Disponível em: <http://seer.ufrgs.br/Movimento/article/view/27495>. Acesso em: 11 jul. 2012.

Recebido em: 25 maio 2012. Aceito em: 11 julho 2012. 